\title{
Improving Residence Time Distribution in Glass Melting Tanks Using Additionally Generated Lorentz Forces
}

\author{
Senan Soubeih ${ }^{1 *}$, Ulrich Luedtke ${ }^{1}$ and Bernd Halbedel ${ }^{2}$ \\ 1. Department of Electrical Engineering and Information Technology, Electrothermal Energy Conversion Group, Technische \\ University Ilmenau, Ilmenau 98684, Germany \\ 2. Department of Mechanical Engineering, Group of Inorganic-Nonmetallic Materials, Technische University Ilmenau, Ilmenau \\ 98684, Germany
}

\begin{abstract}
Continuous glass melting tanks represent thermo-chemical reactors with very complex flow patterns. Controlling the flow patterns within the glass melting tanks with the aim of improving their performance is one of the glass industry primary challenges. The tank performance is basically determined by the RTD (residence time distribution) of the glass melt, which directly impacts the glass quality and energy distribution. In the present work, numerical simulations are carried out on the electromagnetic flow control to investigate how well the flow can be controlled by externally generated electromagnetic (Lorenz) forces that are added to the glass melt. Furthermore, the melting tanks are equipped with supplementary electric heating systems called "electric boosters". The desired result would be an improved RTD. The electromagnetic flow control is called "electromagnetic boosting" and can be realized by exposing the glass bath to an external magnetic field generating Lorentz forces on the glass melt as an additional flow component. The numerical simulations of the present study require coupled calculations of electromagnetic field, flow field, and temperature field, because the material properties of glass melt are strongly temperature-dependent. The computational results show that electromagnetic boosting is an excellent way of improving the RTD in glass melting tanks, ultimately resulting in better glass quality and increased productivity. Of course, the glass industry is highly interested in achieving exactly this result.
\end{abstract}

Key words: Lorentz force, RTD, magnet system, glass melt, numerical simulation.

\section{Introduction}

\subsection{Continuous Glass Melting Tanks with Electric Boosting}

In the glass industry, most furnaces for glass mass production are continuous melting tanks, where the raw materials-batch is continuously fed into the tank-inlet and glass melt flows continually out of the tank-outlet. Such melting tanks have very complex flow patterns, as all the basic processes of melting, degasing (bubble removal), and homogenizing take place in one tank. The batch and melt are mostly heated by natural gas burners in the combustion space above the melt surface. Temperature gradients within

\footnotetext{
*Corresponding author: Senan Soubeih, M.Sc., research field: numerical simulation of electromagnetic flow control. E-mail: senan.soubeih@tu-ilmenau.de.
}

the glass melt between the surface and the bottom cause density differences which lead to free convection flows in the melting tank. The free convections are supported by a wall and/or a bubbling system and/or an electric heating system (Fig. 1) close to the normal hot spot which separates the tank in a melting zone and a fining (degasing and homogenization) zone.

The glass melt requires a MRT (minimum residence time) within the melting tank in order to be adequately processed. Processing includes completely dissolving the raw materials, removing the bubbles, and homogenizing the melt. Therefore, the glass melt must be circulated and mixed sufficiently within the tank to yield a product that meets the quality specifications.

Convection flow has the greatest influence on the residence time as well as the circulating and mixing 


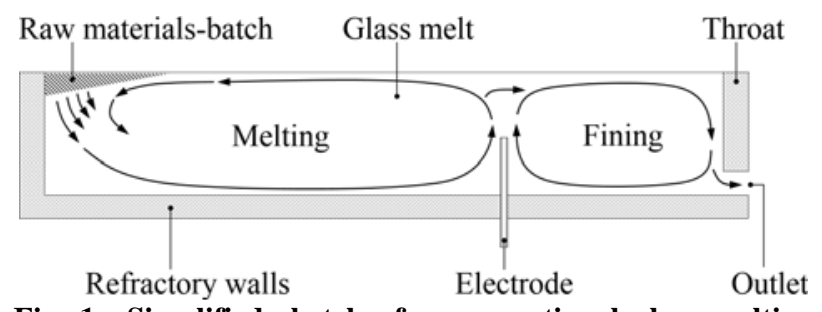

Fig. 1 Simplified sketch of a conventional glass melting tank with bottom boosting electrodes and illustration of the characteristic flow pattern.

behavior of the melt. Thus the processing performance can be improved by intensifying the free convections. One method of doing so is to heat the glass bath locally close to the hot spot using direct electric heating (called electric boosting), creating local convection currents which lift the relatively cold glass melt layers from the bottom to the surface. This supports the flow separation in separate melting and fining zones as illustrated in Fig. 1. For this purpose, rode electrodes of molybdenum are placed in glass melting tanks and then used to conduct electric current through the glass bath. The joule heating caused by the electric current as it passes through the glass melt leads to buoyancy forces due to the density differences. Fig. 1 shows a simplified schematic sketch of a conventional glass melting tank fired with fossil fuels, and equipped with supplementary boosting electrodes installed vertically through the tank bottom. The characteristic flow pattern of the glass melt in a longitudinal cross section of the tank is also illustrated in the sketch.

The boosting electrodes are arranged evenly spaced in a row across the width of the tank. Electric boosting is less effective at the bottom in the spacing areas between the electrodes, where the relatively cold glass melt at the bottom flows directly into the tank-outlet. This impairs the glass quality. The flow of the colder bottom glass melt can be prevented by a barrier wall of refractory materials mounted on the tank bottom behind the boosting electrodes. However, the disadvantage of this method is the refractory corrosion.

Moreover, electromagnetic (Lorentz) forces arise in the vicinity of the electrodes. These forces are called

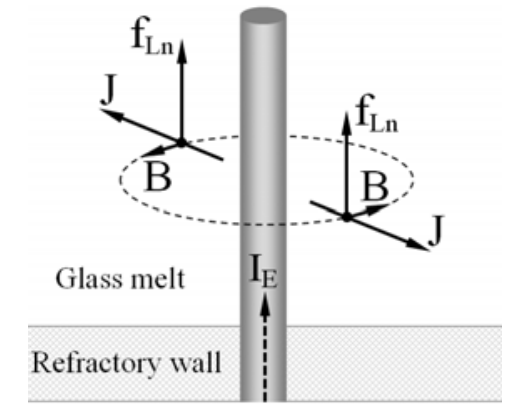

Fig. 2 Natural $f_{L n}$ are generated and surround the electrode.

$I_{E}$ : electrode current, $B$ : induced magnetic flux density "eigenfield", $J$ : electric current density.

natural Lorentz forces $\left(f_{L n}\right)$. Natural Lorentz forces are induced when the electric current density $(J)$ in the glass melt interacts with the magnetic flux density $(B)$ ("eigenfield") induced by the electrode current. Fig. 2 illustrates how $f_{L n}$ are generated and which impact they have. They act towards the electrode top, causing an extra flow component only in the tiny area surrounding the electrode $[1,2]$.

\subsection{Electromagnetic Boosting in Glass Melting Tanks}

The glass industry continually aims to improve the furnace performance, striving for better glass quality and lower energy consumption.

In our work we investigate how additionally imposed Lorentz forces [3] called artificial Lorentz forces $\left(f_{L a}\right)$ can potentially improve the furnace performance. The $f_{L a}$ are generated when the electric current density distribution in the glass melt interacts with the magnetic flux density $\left(B_{c}\right)$ generated by external coils. The external coils are always placed between two electrodes underneath the tank bottom so that the $f_{L a}$ result in the glass melt between the electrodes. If the correct electrical coil current is chosen, then the $f_{L a}$ will run opposite to the main flow direction of the glass melt as illustrated in Fig. 3. The coil current frequency must be the same as the electrode current frequency in order to synchronize the phase alteration so that the $f_{L a}$ always run in the predetermined direction.

This innovative approach is called electromagnetic boosting. It will prevent the flow of the colder bottom 


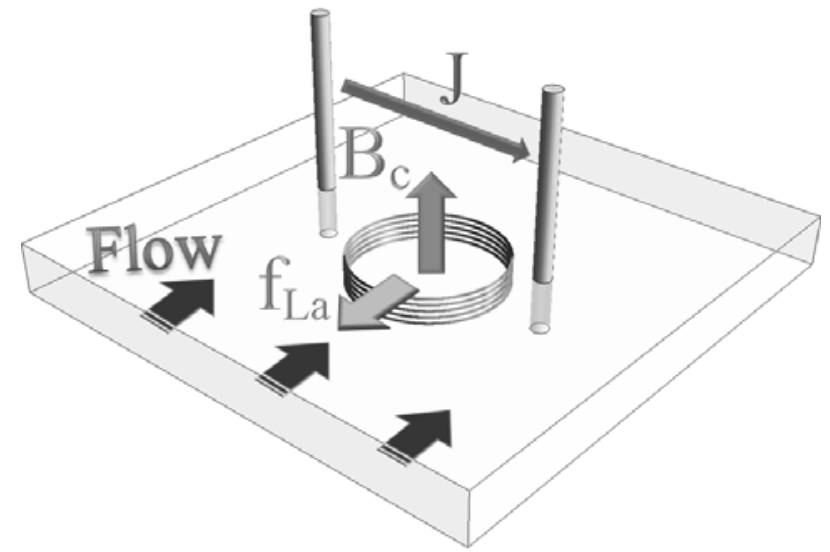

Fig. 3 Physical principle of the $\boldsymbol{f}_{\mathbf{L a}}$.

$J$ : electric current density, $B_{c}$ : magnetic flux density generated by the coil.

glass melt between the electrodes and recirculate it in the melting zone. Electromagnetic boosting is achieved by creating a controllable material-free electromagnetic wall across the width of the tank, with the aim of obtaining an increased MRT, which will in turn enhance the glass quality as well as the tank productivity.

In the numerical investigations the authors seek the optimum process conditions using a tank model which has (a) a simplified tank-inlet for raw materials-batch, (b) a typical electric boosting system consisting of six bottom electrodes, and (c) an electromagnetic boosting system consisting of five coils placed underneath the tank bottom between the electrodes. Fig. 4 shows a schematic sketch of such a tank that is used to produce container glass.

\section{Numerical Simulation}

\subsection{Coupling of Fields}

The numerical simulations of the current problem require coupling electromagnetic field, flow field, and temperature field calculations because the electrical conductivity $\kappa$ and viscosity $\eta$ of molten glass are strongly nonlinearly temperature-dependent, where $\kappa$ increases and $\eta$ decreases with temperature [4].

The local glass melt temperature determines the electrical conductivity value which in turn determines the electric current density distribution as well as the
Lorentz force density distribution. The latter influences the glass melt flow which of course has an important impact on the temperature distribution within the glass melt. Fig. 5 shows schematically how the fields are coupled.

\subsection{Magnetic Field Calculation with FLUENT}

In the simulations the temperature-dependent electrical conductivity value must be updated in the solution domain, and the electric current and Lorentz force densities must be recalculated. Thus, coupled calculations of a three-dimensional problem using two different simulation tools would cause a gigantic effort.

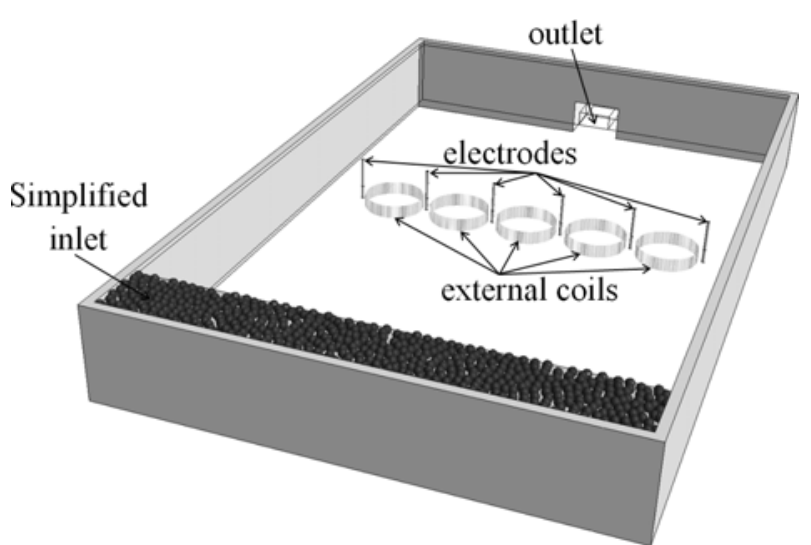

Fig. 4 Simplified view of the glass melting tank with an electromagnetic boosting system comprised of 6 electrodes and 5 intermediate coils placed in the thermal insulation of the tank bottom.

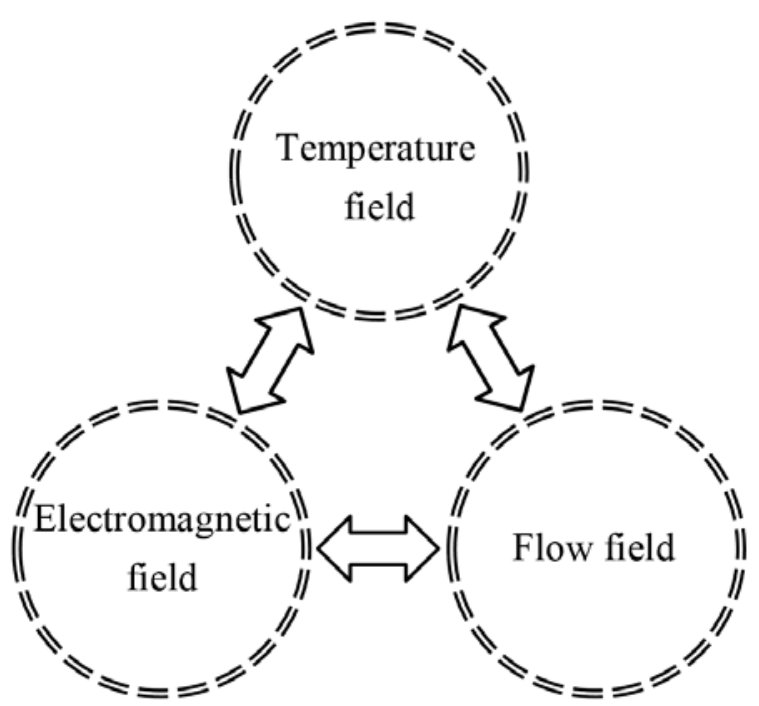

Fig. 5 Interaction of the three fields. 
FLUENT is very well suited to calculate flow (Navier-Stokes equations: velocity and pressure) and temperature distribution using the finite volume method. The idea is to include the magnetic field calculation completely in FLUENT using the so-called UDF (user defined functions) and the UDS (user defined scalars), Eq. (1):

$$
\begin{gathered}
\frac{\partial \rho \phi_{k}}{\partial t}+\frac{\partial}{\partial x_{i}}\left(F_{i} \phi_{k}-\Gamma_{k} \frac{\partial \phi_{k}}{\partial x_{i}}\right)=S_{\phi_{k}} \\
k=1, \ldots, N_{\text {scalars }}
\end{gathered}
$$

FLUENT is able to solve up to 50 diffusion equations, storing the results in a scalar field $\phi_{k}$ [5].

Only one simulation tool will be running to efficiently solve this coupled nonlinear three-dimensional problem.

The temperature dependency of electrical conductivity $\kappa$, dynamic viscosity $\eta$ and mass density $\rho$ of the glass melt is considered in our simulations. All other material properties are assumed to be constant.

Basic equations for the electromagnetic field calculation are Eqs. (2)-(5):

$$
\begin{gathered}
\text { curl } \vec{E}=-\frac{d \vec{B}}{d t}, \text { (Faraday's law) } \\
\text { curl } \vec{H}=\vec{J}+\frac{d \vec{D}}{d t}, \text { (Maxwell-Ampere law) } \\
\operatorname{div} \vec{J}=0,(\text { continuity of current) } \\
\vec{J}=\kappa(\vec{E}+\vec{v} \times \vec{B}),(\text { Ohm's law) }
\end{gathered}
$$

Generally the authors assume for the magnetic vector potential $\vec{A}$, described with Eqs. (6) and (7):

$$
\begin{array}{r}
\vec{B}=\operatorname{curl} \vec{A} \\
\operatorname{div} \vec{A}=0
\end{array}
$$

In a final step the mathematical law (8) is needed for continuously differentiable vector functions:

$$
\text { grad div } \vec{A}-\text { curl curl } \vec{A}=\operatorname{div} \text { grad } \vec{A}
$$

Usually the current is an alternating current with a frequency of $50 \mathrm{~Hz}$. For this low frequency the derivative of $\vec{D}$ in the Maxwell-Ampere law (3) can be neglected. Furthermore, the electrical conductivity of the glass melt is very low, which is why the authors neglect the induced currents. Subsequently, the derivative of $\vec{B}$ in Faraday's law (2) can be neglected as well. Furthermore, the glass melt has a very low velocity of maximally $10 \mathrm{~mm}$ per second, therefore we can neglect the $\vec{v} \times \vec{B}$ term in (5) [6]. To achieve a homogeneous distribution of heat sources in the glass melt between the electrodes we apply a constant electric potential of a three-phase current system. The authors apply an adapted phase for each electrode, making it necessary to use complex values to describe the electromagnetic field. First we calculate the electrical current in the electrodes and within the glass melt using the complex scalar potential $\varphi$ with Eq. (9):

$$
\operatorname{div} \kappa \operatorname{grad} \underline{\varphi}=0 \text {, with } \operatorname{grad} \underline{\varphi}=-\frac{\vec{J}}{\kappa}
$$

Assuming a constant permeability $\mu=\mu_{0}$, the authors calculate the magnetic field $\underline{\vec{B}}$ using the well-known magnetic vector potential $\underline{\vec{A}}$ as follows:

$$
\operatorname{div} \operatorname{grad} \underline{\vec{A}}=-\mu \underline{\vec{J}}
$$

The four complex field quantities $\underline{\varphi}, \underline{A}_{\gamma}, \underline{A}_{y}$, and $\underline{A}_{z}$ are fragmented into eight components and solved using eight UDS diffusion equations. The so-called UDF are used to define the sources and temperature-dependent material properties. By solving (9) and (10) we are able to calculate the magnetic flux density $\underline{\vec{B}}$ ("eigenfield") induced by the electrode current $\overrightarrow{\underline{I}}_{E}$. This magnetic flux density ("eigenfield") together with the electric current density within the glass melt leads to the natural Lorentz forces (Fig. 2). The additional (artificial) Lorentz forces are generated by the magnetic flux density $\underline{\vec{B}}_{c}$ of the external coils in interaction with the electric current density $\underline{\vec{J}}$ within the glass melt (Fig. 3). The magnetic flux density of the coil $\underline{\vec{B}}_{c}$ (external field) is calculated by the Biot-Savart law (11) caused by an imposed complex current $\vec{I}_{c}$ in the external coils: 


$$
\underline{\vec{B}}_{c}(\vec{r})=\frac{\mu_{0}}{4 \pi} \int_{l} \frac{\overrightarrow{\vec{I}}_{c}\left(\vec{r}^{\prime}\right) \times\left(\vec{r}-\vec{r}^{\prime}\right)}{\left|\vec{r}-\vec{r}^{\prime}\right|^{3}} d l^{\prime}
$$

This is an analytical solution for a domain with a constant permeability.

Finally, we can calculate the time-averaged part of the total Lorentz force density $\vec{f}_{l}$ in the glass melt needed for the flow calculation as follows:

$$
\vec{f}_{l}=\operatorname{Re}\left(\underline{\vec{J}} \times\left(\operatorname{curl} \underline{\vec{A}}+\underline{\vec{B}}_{c}\right)^{*}\right)
$$

\section{Results and Discussion}

\subsection{Impact of the RTD (Residence Time Distribution)}

In the investigation the authors aim to achieve the optimal furnace performance. Electromagnetic boosting can improve the furnace performance in two different ways: (1) improving the initial glass quality while maintaining the initial tank productivity, or (2) increasing the initial tank productivity while maintaining the initial glass quality.

To investigate the achievable glass quality, it is very important to study the RTD. The RTD describes the length of time each glass particle spends inside the tank, since glass particles that follow different trajectories will require more or less time to pass through the tank.

For an accurate prediction of the RTD in our simulation, 20,000 massless particles are tracked beginning at the tank inlet using a high order runge-kutta tracking scheme $[7,8]$.

\subsection{Improving the Glass Quality}

A systematic study is carried out to obtain the highest MRT which is the shortest amount of time a glass particle spends inside the tank [9]. Therefore, the flow patterns of many different cases are simulated using the glass melting tank model with a simplified tank-inlet, six boosting electrodes, and five external coils. A constant mass flow rate of soda-lime glass with a constant temperature is assumed at the tank-inlet for all cases. We fix the electric voltage at the electrodes in all cases $\left(U_{E}=57 \mathrm{~V}\right)$. Only the coils current $\left(I_{c}\right)$ is systematically increased from $0 \mathrm{~A}$, which represents the initial case (without electromagnetic boosting), up to $1,400 \mathrm{~A}$ with a maximum interval of $200 \mathrm{~A}$ and a minimum interval of 25 A. Thus the intensity of the artificial Lorentz force is raised systematically.

Fig. 6 depicts the resulting MRT as the $I_{c}$ changes. The maximal increase obtained for MRT is about $82.4 \%$, attained when the coil current value is at 425 A. A higher coil current value leads to a lower MRT. It thus becomes obvious that the optimal electromagnetic boosting performance, assuming predetermined glass composition and electrode current, requires a specific coils current value.

Fig. 7 shows the cumulative RTD of the cases with $\left(I_{c}=425 \mathrm{~A}\right)$ and without $\left(I_{c}=0\right)$ electromagnetic boosting. The cumulative RTD, also called the $\mathrm{F}(\mathrm{t})$ curve, can be depicted by plotting the mass fraction rise from zero to unity at the tank-outlet as a function of time ( $\mathrm{t}$ ). Both cases are compared with the ideal case, which assumes that all particles introduced into the tank-inlet at $t=0$ will leave the tank after the mean residence time, where the consumed energy would be evenly distributed to all particles. The mean residence time $\bar{t}$ is given by the ratio of the tank capacity (volume $V$ of the glass bath) to the volumetric flow rate $v[10,11]$ :

$$
\bar{t}=\frac{V}{v}
$$

A very important descriptive parameter of the RTD is the spread $\sigma$ which measures the deviation amount from the mean residence time $\bar{t}$ :

$$
\sigma=\sqrt{\frac{1}{n} \sum_{i=1}^{n}\left(t_{i}-\bar{t}\right)^{2}}
$$

where, $n$ is the total number of the tracked particles and $t_{i}$ is the residence time of each particle $i$.

A lower spread indicates that the RTD tends to be closer to the ideal case, where the spread of the ideal case is equal to zero, since $t_{i}=\bar{t}$. The electromagnetic 


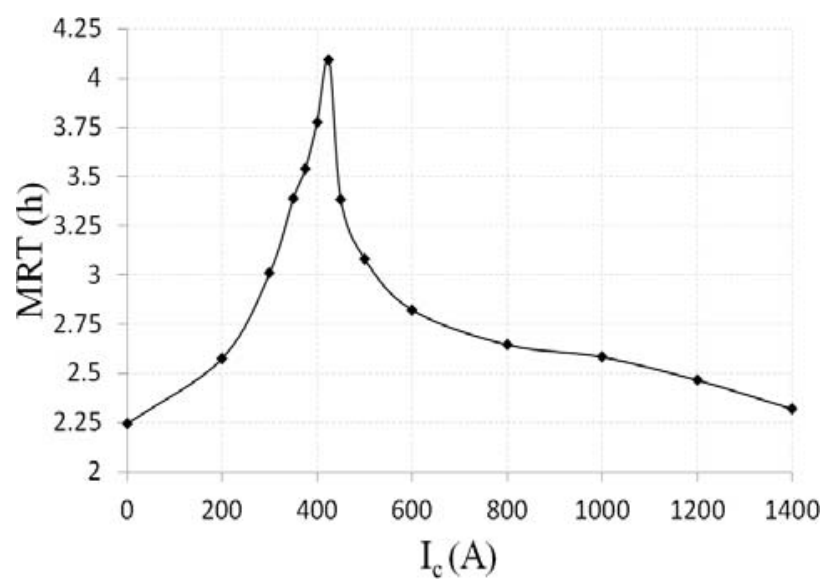

Fig. 6 The MRT as a function of the $I_{c}$. $U_{E}=57 \mathrm{~V}, \quad{ }_{0}=1.157 \mathrm{~kg} / \mathrm{s}$, soda-lime glass.

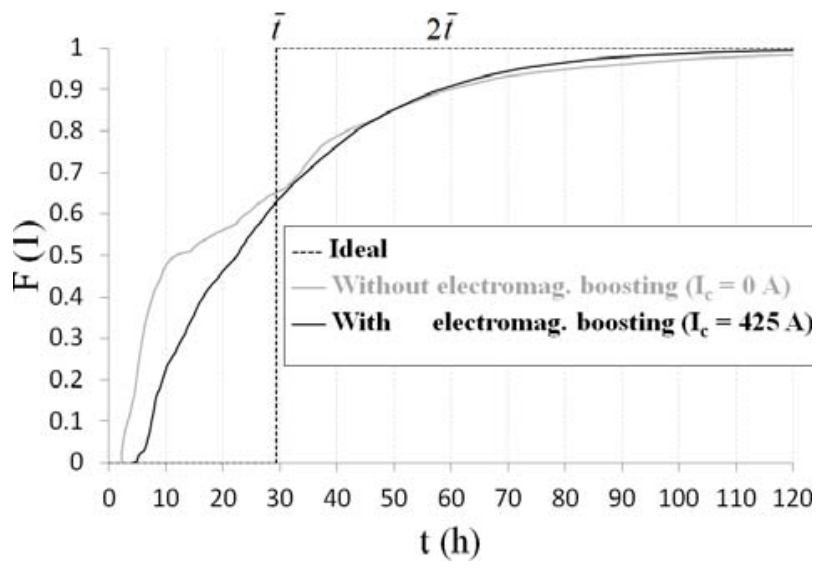

Fig. 7 Comparison of the cumulative RTD or $F(t)$ curves for the simplified glass melting tank in Fig. 4 with and without electromagnetic boosting.

boosting (at a coil current of $I_{c}=425 \mathrm{~A}$ ) reduces the spread by about $27 \%$ and brings the RTD closer to the ideal distribution.

The comparison of the $\mathrm{F}(\mathrm{t})$ curves reveals that without electromagnetic boosting, about $15 \%$ of the glass particles have a residence time lower than the MRT of those glass particles with electromagnetic boosting at $I_{c}=425 \mathrm{~A}$. These particles with very short residence times have lower glass quality.

Furthermore, in the time domain where $t<\bar{t}$, electromagnetic boosting allows fewer particles to leave the tank, so that the glass particles will have a longer processing time. This has a positive effect on the glass quality. On the other hand, if the time domain exceeds twice the mean residence time $t>2 \bar{t}$, electromagnetic boosting allows more particles to leave the tank, thus decreasing unnecessary processing.

As a result, the timed distribution of the consumed energy is more homogeneous and has a narrower spread, so that the glass quality as well as the melting process efficiency is enhanced.

\subsection{Increasing the Tank Productivity}

Increasing the tank productivity means increasing the mass flow rate through the tank. In other words, the aim is to produce more glass mass per time unit.

Under the initial process conditions of the glass melting tank $\left(I_{c}=0\right)$, increasing the glass mass flow rate would result in an MRT shorter than required to adequately process the raw materials. Consequently, the resulting product would not satisfy the quality standards. By applying electromagnetic boosting $\left(I_{c}=\right.$ $425 \mathrm{~A})$, it will be possible to maintain the required glass quality (the required MRT) while increasing the mass flow rate through the tank.

To investigate the potential of increasing the productivity by using electromagnetic boosting, the mass flow rate at the tank-inlet is systematically increased from the initial value $\dot{m}_{0}$ up to $4.5 \dot{m}_{0}$. This theoretical mass flow rate increase is carried out under the impact of electromagnetic boosting at $I_{c}=425 \mathrm{~A}$ which offers the highest MRT.

Fig. 8 shows the resulting MRT after the mass flow rate increase as $\dot{m} / \dot{m}_{0}$. The increased MRT was returned to the initial value $M R T_{0}$ with a mass flow rate that equals four times the initial mass flow rate $\dot{m}$ $=4 \dot{m}_{0}$. A greater mass flow rate increase results in less than the required $M R T_{0}$.

To compare the spreads $\sigma$ of the considered cases we compare the ratios $\sigma / \bar{t}$ of each case, since each case has a different mean residence time $\bar{t}$. All cases with electromagnetic boosting are characterized by a lower ratio value $\sigma / \bar{t}$ as compared to the initial case (without electromagnetic boosting). Clearly, the consumed energy is better distributed. Furthermore, the glass quality is better when the MRT is kept 
higher than $M R T_{0}$.

Fig. 9 shows the resulting $F(t)$ curve after increasing the mass flow rate to $4 \dot{m}_{0}$ under the impact of electromagnetic boosting $\left(I_{c}=425 \mathrm{~A}\right)$ in comparison with the $\mathrm{F}(\mathrm{t})$ curve of the initial mass flow rate $\dot{m}_{0}$ without electromagnetic boosting $\left(I_{c}=0\right)$. The MRT of the case with electromagnetic boosting at $4 \dot{m}_{0}$ is equal to $M R T_{0}$. The required glass quality is given even though the glass mass per time unit is increased fourfold.

An additional important advantage is that the RTD of the case with electromagnetic boosting at $4 \dot{m}_{0}$ has a much lower spread and tends to be closer to the ideal distribution, resulting in an improved melting process and a better glass quality.

To better explain the potential of electromagnetic boosting at $I_{c}=425 \mathrm{~A}$, let us view the case at $2 \dot{m}_{0}$

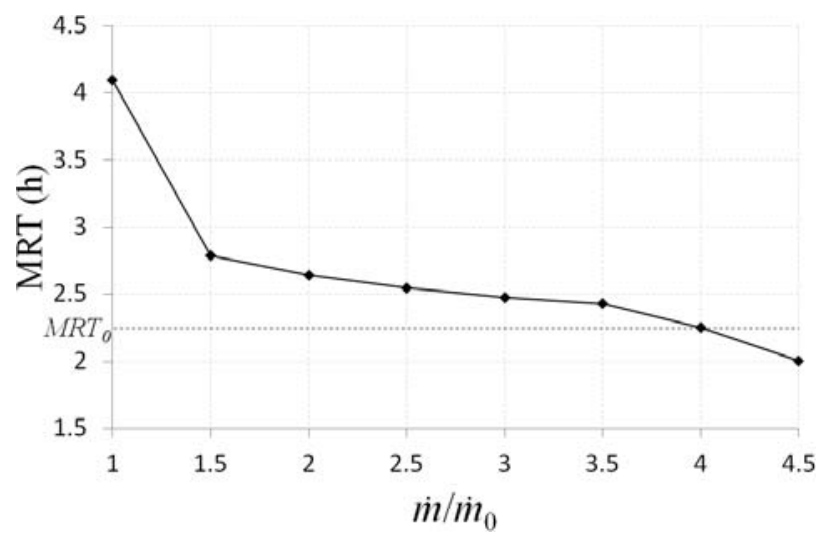

Fig. 8 MRT dependence on mass flow rate $\dot{m} / \dot{m}_{0}$ with electromagnetic boosting at $I_{c}=425 \mathrm{~A}$ and $U_{E}=57 \mathrm{~V}$.

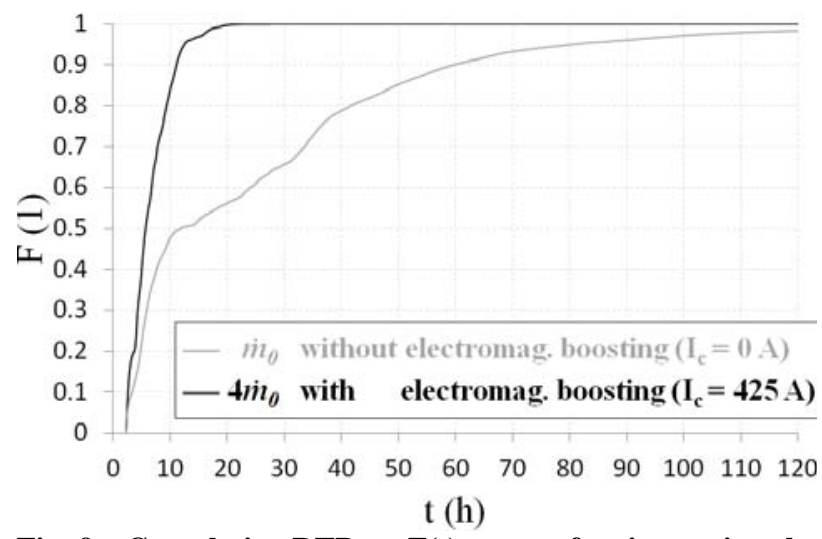

Fig. 9 Cumulative RTD or $F(t)$ curve after increasing the mass flow rate four times with $I_{c}=425 \mathrm{~A}$ in comparison with the initial situation $\left(\dot{m}=\dot{m}_{0}, I_{c}=0\right)$.

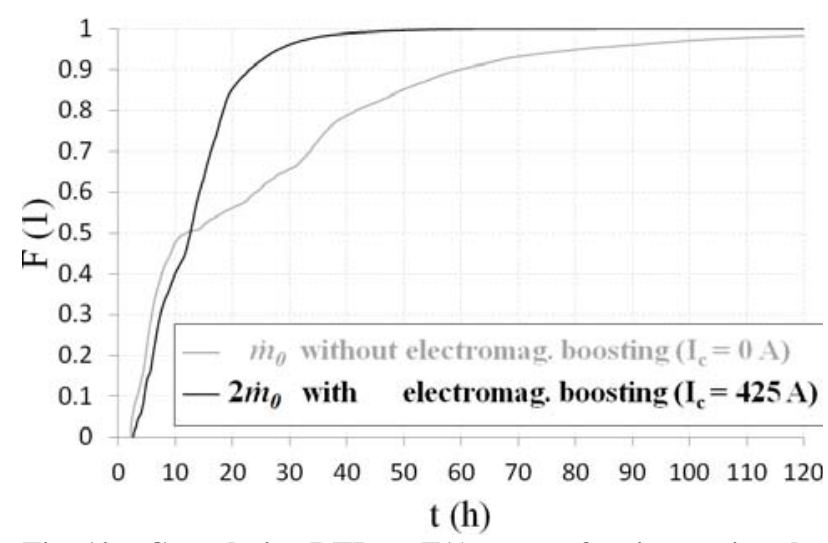

Fig. 10 Cumulative RTD or $F(t)$ curve after increasing the mass flow rate two times at $I_{c}=425 \mathrm{~A}$ in comparison with the initial situation $\left(\dot{\boldsymbol{m}}=\dot{\boldsymbol{m}}_{0}, I_{c}=\mathbf{0}\right)$.

Table 1 Evaluation of the furnace productivity with $\left(I_{c}=\right.$ $425 \mathrm{~A})$ and without $\left(I_{c}=0\right)$ electromagnetic boosting.

\begin{tabular}{|c|c|c|c|c|}
\hline \multirow{3}{*}{ No. } & \multirow{3}{*}{ Case } & $\dot{m}$ & $M R T$ & $\sigma$ \\
\hline & & $\overline{\dot{m}_{0}}$ & $\overline{M R T_{0}}$ & $\overline{\bar{t}}$ \\
\hline & & {$[1]$} & {$[1]$} & [1] \\
\hline 0 & Initial situation & 1 & 1 & 1.081 \\
\hline 1 & \multirow{3}{*}{$\begin{array}{l}\text { With electromagnetic } \\
\text { boosting }\left(I_{c}=425 \mathrm{~A}\right)\end{array}$} & 1 & 1.824 & 0.770 \\
\hline 2 & & 2 & 1.178 & 0.566 \\
\hline 3 & & 4 & 1 & 0.569 \\
\hline
\end{tabular}

which has a lower spread and a higher MRT than the initial case. In Fig. 10 the F (t) curves are compared. In the case with electromagnetic boosting $\left(I_{c}=425 \mathrm{~A}\right)$ at $2 \dot{m}_{0}$ the MRT is increased by $17.8 \%$, which enhances the glass quality despite the doubled mass flow rate.

Table 1 summarizes the MRT comparisons of the spreads $\sigma$ before and after electromagnetic boosting. We start at $\dot{m}=\dot{m}_{0}$ without electromagnetic boosting $\left(I_{c}=0\right)$ and follow up with electromagnetic boosting $\left(I_{c}=425 \mathrm{~A}\right)$ at $\dot{m}=\dot{m}_{0}, \dot{m}=2 \dot{m}_{0}$, and $\dot{m}=4 \dot{m}_{0}$.

\section{Conclusions}

The numerical study with the commercial tool FLUENT on a simplified model of a real glass melting tank shows that it is possible to use externally generated Lorentz forces to control the flow patterns in glass melting tanks, significantly improving the RTD. As a direct result, the energy distribution within the glass melt is greatly improved, yielding a more 

Additionally Generated Lorentz Forces

homogeneous and thus higher quality final glass product while increasing the processing efficiency.

Electromagnetic boosting is a new and innovative approach for sophisticated glass melting tanks. Installing an electromagnetic boosting system is a viable investment in order to improve the operational flexibility of the glass tank

The present work is the foundation necessary to convert the idea into an effective industrial process.

\section{Acknowledgments}

This work is supported by the Freistaat Thüringen and by the Förderkreis Elektrowärme Ilmenau e.V.

\section{References}

[1] Choudhary, M. K. 1995. "In a Modeling Study of Flow and Heat Transfer in the Vicinity of an Electrode." Proceedings of the XVIIst International Congress on Glass 6: 100-7.

[2] Hofmann, O. R., and Philipp, G. 1992. "Importance of Lorentz Force in Electrically Heated Glass Melts." Glastech. Ber. 65 (5): 142-9.

[3] Hülsenberg, D., Halbedel, B., Conrad, G., Thess, A., Kolesnikov, Y., and Lüdtke, U. 2004. "Electromagnetic
Stirring of Glass Melts Using Lorentz Forces-Experimental Results." Glass Sci. Technol. 77 (4): 186-93.

[4] Cepite, D., Jakovičs, A., Halbedel, B., and Krieger, U. 2007. "Modeling of Electromagnetic Glass Convection with Temperature-dependent Properties of the Melt." Magnetohydrodynamics 43 (2): 195-204.

[5] ANSYS, Inc. 2011. FLUENT 14.5 Documentation.

[6] Krieger, U., Halbedel, B., Hülsenberg, D., and Thess, A. 2008. "Electromagnetic Effects on Glass Melt Flow in Crucibles.” Eur. J. Glass Sci. Technol. A 49 (1): 33-40.

[7] Beerkens, R. G. C. 2002. "Melting and Fining: Modeling of the Melting Process in Industrial Glass Furnaces." In Mathematical Simulation in Glass Technology, Schott Series on Glass and Glass Ceramics 17-72.

[8] Eisermann, H., Lange, U., Loch, H., and Weidmann, G. 2002. "Homogenizing and Conditioning: The Intensity of Mixing Processes." In Mathematical Simulation in Glass Technology, Schott Series on Glass and Glass Ceramics, 165-92.

[9] Kuhn, W. S., Moukarzel, C., and Clodic, D. 2002. "In Some Aspects to the Minimum Residence Time in Glass Tanks and its Mathematical Modeling." Proceedings of the 2002 glass odyssey: 6th ESG Conference.

[10] Hlaváć, J. 1983. The Technology of Glass and Ceramics: An Introduction. Amsterdam: Elsevier.

[11] Levenspiel, O. 1999. Chemical Reaction Engineering. New York: Wiley. 